\title{
MEDIO AMBIENTE FETAL
}

COORDINADOR:

\author{
Dr. Jorge E. Medina Murillo
}

INSTITUTO MATERNO INFANTIL

\section{Dr. Arturo Rodríguez Soto \\ Dr. Alvaro Velasco Chiriboga}

HOSPITAL SAN JOSE

Dr. Hugo Buriticá

CLINICA SAN PEDRO CLAVER

Dr. Daniel Rodríguez

HOSPITAL SAN IGNACIO

Dr. Julián Bustillo Yepes

Dr. Manuel José Palau

HOSPITAL MILITAR CENTRAL

\section{Dr. Uriel Acero Rodríguez}

Dr. Hermann Uribe Correa

HOSPITAL INFANTIL

Dr. Germán Marín Ocampo

Dr. Jaime Enrique Ruiz Acevedo

* Relato Oficial.

SOCIEDAD COLOMBIANA DE OBSTETRICIA Y GINECOLOGIA - $\times$ CONGRESO NACIONAL DE OBSTETRICIA Y GINECOLOGIA — Cali - Diciembre 4-8 - 1973.

\section{Primera Parte}

En los últimos 10 años todos los esfuerzos de la Obstetricia se han encaminado a estudiar el feto en desarrollo, el líquido amniótico y la unidad fetoplacentaria, tratando de identificar el producto de alto riesgo para obtener individuos de óptimas condiciones físicas y mentales.

El estudio del medio ambiente fetal va a tener entonces como meta final la disminución de los índices de morbilidad y mortalidad perinatal. Es necesario en consecuencia identificar y prevenir las causas de morbilidad y mortalidad perinatales; en este empeño apareció el concepto de alto riesgo, al cual nos vamos a referir someramente.

Gold (47) en 1968 define el alto riesgo en salud materno infantil como la probabilidad grave o muy importante de morbilidad 0 mortali- 
dad para la madre, el feto o el neonato.

Nesbitt (89) en una extensa experiencia en problemas de morbilidad perinatal, ideó un sistema de identificación del riesgo obstétrico, considerando numerosos factores; en 1964 se inició su aplicación en el Departamento de Obstetricia y Ginecología de la Universidad de Nueva York.

Marmol y cols. (76) utilizando un sistema similar al de Nesbitt, encuentran que el $70 \%$ de mortalidad materna ocurre en mujeres clasificadas como de alto riesgo.

Goodwin y cols. (48) describen un sistema de puntaje que incluye factores de riesgo identificables en las etapas tempranas de la gestación, en el preparto y en la iniciación del trabajo.

Rantakallio (111) en 1966 en el norte de Finlandia, analizó 12.000 partos, investigando las características biológicas de la madre, su historia obstétrica y sus condiciones socio-económicas, encontró que el $14 \%$ de la población estudiada debía ser considerada como de alto riesgo.

En nuestro medio, Cobo y cols. (21) y el grupo de la Universidad Javeriana utilizando como base los esquemas de Nesbitt y Gold, propusieron un sistema de registro en el cual se tienen en cuenta factores patológicos y socio-económicos propios de nuestro país. Hoy en todas nuestras instituciones la identificación del embarazo con alto riesgo es imprescindible, ya que va a condicionar una asistencia óptima para la embarazada brindándole todos los recursos de salud, avances científicos y tecnológicos buscando así disminuir la morbilidad y la mortalidad perinatal.
Es importante destacar, que varios hechos en el curso de la gestación pueden guardar relación con la morbilidad y mortalidad perinatal. Entre ellos se cuentan complicaciones del embarazo (toxemia, placenta previa, desprendimiento de placenta), complicaciones del parto (presentaciones distócicas, distocias dinámicas, -sufri miento fetal), y enfermedades materno-fetales (isoinmunización, diabetes, anemia, etc.) y que hay datos que hacen resaltar el hecho de que la cantidad y la calidad de la asistencia prenatal, así como la adecuada clasificación del riesgo obstétrico van a influir en la mortalidad perinatal.

La preocupación existente en todos los sitios por la evaluación adecuada del riesgo obstétrico y fetal, se ha visto recompensada por una notoria disminución de los índices de morbilidad y mortalidad perinatales.

En Estados Unidos en el período de 1945 a 1964, ha disminuído la mortalidad perinatal en un $28.6 \%$.

Actualmente se lleva a cabo en Estados Unidos un Proyecto Cooperativo Perinatal de alcance nacional, en el cual se estudian 60.000 gestaciones y el resultado de las mismas.

Los niños productos de estas gestaciones se están controlando y algunos han alcanzado la edad de 8 años. Entre las observaciones hechas hasta la fecha son importantes de destacar, que en niños prematuros hay aumento importante de anomalías neurológicas y que la anoxia y la asfixia son factores etiológicos frecuentes de daño cerebral (144).

La magnitud del problema de los daños sobre tejido nervioso se ilustra por cálculos del siguiente número de niños impedidos en Estados Unidos para 1970: 2'624.000 retardados mentales; 437.000 con parálisis 
cerebral y 428.000 con epilepsia (141).

Definimos como alto riesgo obstétrico a todos los factores biológicos, psíquicos y ambientales que de alguna manera puedan alterar la evolución normal del embarazo, parto o puerperio, incidiendo en la madre, el feto $\circ$ el recién nacido.

El niño con alto riesgo ha sido definido como aquel que tiene probabilidad de presentar un impedimento físico, intelectual, social o de la personalidad que puede dificultar el crecimiento y el desarrollo normales y la capacidad para aprender. Este impedimento puede haberse originado en período prenatal, perinatal o postnatal y pudiera resultar de influencias hereditarias o ambientales desfavorables, actuando por separado o en combinación (47).

En la actualidad los parámetros clínicos corrientes son inadecuados para descubrir el feto de riesgo alto. $\mathrm{Se}$ requiere el estudio de las modificaciones del medio fetal que preceden al trastorno clínico.

Todos los elementos que se estudian actualmente para la valoración del medio fetal pueden ayudar a descubrir trastornos incipientes, a determinar la mejor época para la vida fetal extrauterina y a disminuir la mortalidad y la morbilidad infantiles $y$ perinatales.

\section{VALORACION DEL MEDIO AMBIENTE FETAL}

El control prenatal, es el medio más adecuado y fácil para valorar el desarrollo fetal. Hoy día tiene gran importancia establecer en la consulta prenatal el grado de riesgo maternofetal, con el fin de seleccionar adecuadamente, aquellos casos de alto riesgo, para un estudio y seguimien- to meticuloso durante todo el embarazo, que nos permita llevar a feliz término, el producto de la concepción. Kohl (65) informa una reducción de mortalidad perinatal de $16,6 \%$ para pacientes sin control prenatal, a $1,3 \%$ en pacientes con ocho o más controles.

Hoy día contamos con una variedad de métodos paraclínicos, que nos permiten estudiar correctamente el medio ambiente fetal. Tales procedimientos son:

1. Radiológicos: Desde el advenimiento de los Rayos $X$ en 1895, han prestado invaluable ayuda en obstetricia, principalmente en el diagnóstico de embarazo múltiple, desproporción céfalo-pélvica, diagnóstico de muerte fetal, malformaciones fetales, embarazo abdominal, presentaciones anómalas y madurez fetal.

En los últimos años se han desarrollado nuevos métodos, como la angiografía, flebografía y amniografía (137).

Sin embargo, no hay que olvidar los riesgos de las radiaciones ionizantes, los cuales están relacionados con la cantidad de radiación, tiempo de exposición y estado del desarrollo embrionario o fetal (27). Algunos autores han Ilamado la atención sobre posibles efectos leucemizantes en niños que habian recibido irradiaciones durante su vida intrauterina $(91,115)$, debido a que durante esta época se puede lesionar el tejido eritropoyético.

Existen pruebas de que el período de mayor susceptibilidad está en la etapa de organogénesis. Teniendo en cuenta estos riesgos, debe limitarse el empleo de procedimientos radiológicos durante la gestación a lo estrictamente necesario (115, 137). 
2. La electrocardiografía fetal, fue empleada por primera vez por Cremer en 1906, utilizando métodos indirectos, por medio de electrodos colocados en la pared abdominal materna, procedimiento relativamente simple; tiene el inconveniente de presentar trazo simultáeno con el corazón materno, lo cual a veces hace difícil la interpretación y evaluación de los complejos electrocardiográficos fetales. Se ha empleado para el diagnóstico de vida fetal temprana, identificación de embarazos múltiples y posibles anomalías cardíacas fetales (106).

3. El ultrasonido fue introducido en obstetricia y ginecología en 1958 por Donald, Macvicar y Brown. Es un método sencillo, completamente inocuo, rápido, que puede repetirse cuantas veces sea necesario sin ningún problema materno fetal. El ultrasonido está basado en ondas de alta frecuencia de sonido que al pasar por las superficies de los tejidos del cuerpo humano se reflejan parcialmente de acuerdo con la densidad del tejido; el sonido reflejado se registra en forma de eco en un tubo de rayos catódicos, como puntos luminosos en una pantalla llamada ecograma bidimencional.

Sus principales indicaciones son el diagnóstico temprano de gestación, diagnóstico diferencial entre útero grávido y tumor ovárico, diferencial de mola hidatiforme, embarazo gemelar, polihidramnios, anencefalia, desproporción cefalopélvica y madurez fetal (124, 138, 139).

El cardioscopio ultrasónico Doppler, se utiliza para detectar vida fetal temprana, 8a a 10 a semana de gestación; ayuda en el diagnóstico diferencial de mola, aborto, desprendimiento de placenta o muerte fetal $(14,62,106)$.
Es el sistema, que más cerca está de cumplir los requisitos para la vigilancia continua automática del estado fetal, durante el trabajo de parto $(62)$.

4․ La monitoría del corazón fetal durante el trabajo de parto, evalúa las condiciones inmediatas intrauterinas y nos muestra las posibilidades de sufrimiento fetal (106).

\section{5․ INDICES ENZIMATICOS DEL MEDIO FETAL}

Tres enzimas plasmáticas maternas se han estudiado primordialmente: la diamino-oxidasa, la fosfatasa alcalina y la oxitocinasa.

La presencia de diamino-oxidasa en la placenta humana fue comprobada por primera vez por Danforth y Gorham en 1935. En 1938 Marcou (79) observó aumento importante en la sangre de las embarazadas. Su uso clínico en el curso de la gestación se vio limitado por la falta de sensibilidad de los métodos de laboratorio. Los utilizados actualmente por Okuyama y Kovayashi (98) han hecho revivir el interés por investigar la concentración plasmática de esta enzima.

La diamino-oxidasa (DAO) es una enzima que participa en la degradación de la histamina, la cual se produce en tejidos en crecimiento, como trofoblasto, tumores malignos, infecciones agudas o heridas en cicatrización. La concentración plasmática en varones y mujeres no embarazadas es similar encontrándose cifras que oscilan entre 2 y 5 unidades. Durante la gestación el organismo materno estimulado por la presencia de la unidad fetoplacentaria, presenta un aumento progresivo hasta la semana vigésima, después de la cual tiende a estabilziarse con cifras por encima de 
500 unidades por cc. de plasma. Su descenso por debajo de estas cifras indica falta del estímulo fetal o falla en la síntesis placentaria. Sus determinaciones deben hacerse en forma seriada. Sus principales indicaciones son: diagnóstico de embarazo, aborto fallido, amenaza de aborto, aborto habitual, antecedentes de muerte fetal y diabetes. También está indicada en el control de tratamientos hormonales durante el embarazo $(98,127,128,149)$.

La fosfatasa alcalina termoestable isoenzima de la fosfatasa alcalina está presente en el suero materno de la gestante, aumenta progresivamente durante el embarazo normal, y es producida en la placenta por el sincitio trofoblasto (129).

Lee (71), Levine (72) y Messer (82) han contribuído con estudios profundos a dilucidar aspectos importantes de esta isoenzima.

Se ha utilizado como índice de función placentaria, y también como índice de madurez fetal (72).

Oxitocinasa. Enzima producida en el sincitio trofoblasto y cuya función primordial es inhibir la actividad de la oxitocina. Fekete en 1930 descubrió la propiedad que tenía el plasma de la embarazada de inactivar la oxitocina.

En 1941 Werley y cols. demostraron que este fenómeno se debía a una enzima presente en el suero de la mujer embarazada y le dieron el nombre de oxitocinasa.

La oxitocinasa aumenta durante la gestación entre 50 y 100 veces, disminuyendo en el embarazo a término a un valor promedio de 5 unidades.

Sus determinaciones deben hacerse también en forma seriada, y se han descrito concentraciones elevadas en inercia uterina prolongada, embarazo múltiple y algunos casos de embarazos prolongados y descenso en casos de insuficiencia placentaria $(15,80$, 136, 149).

\section{6: INDICES ENDOCRINOS DOSIFICACIONES HORMONALES}

\section{Estriol}

El estriol urinario es considerado como uno de los mejores índices para valorar la unidad feto-placentaria $(34,36,51,84)$.

La excreción va aumentando progresivamente durante el embarazo, debido a que las suprarrenales del feto elaboran estrógenos al igual que la placenta, los cuales metabolizados y conjugados por el feto llegan a la madre por la circulación umbilical y son excretados por la orina de la mujer. Esto ha sido apoyado por las observaciones de Diczfalusy quien comprobó que pulmón, hígado, intestino y riñones del feto poseen las facultades de metabolizar y conjugar estrógenos (34).

Se ha demostrado que niveles por encima de $12 \mathrm{mg}$. en 24 horas están asociadas a baja mortalidad perinatal, la cual aumenta cuando los niveles descienden por debajo de $8 \mathrm{mg}$. en 24 horas encontrándose que valores de 3 a $4 \mathrm{mg}$. en 24 horas son indicativos de muerte fetal (49, 50, $51,52,53,130)$.

El estriol sanguíneo se presenta en concentraciones 1.000 veces inferiores a las encontradas en la orina, lo cual hace que este procedimiento sea empleado únicamente en centros altamente especializados en investigaciones endocrinas (113).

En el líquido amniótico también se ha estudiado el estriol para diagnós- 
tico de sufrimiento fetal. Concentraciones inferiores a 100 microgramos indican peligro para la vida fetal. En algunos casos de eritroblastosis se encuentra elevado debido a una sobrecarga de las suprarrenales fetales (16).

\section{GONADOTROPINA CORIONICA HCG}

Se ha utilizado desde 1940 como prueba de embarazo y de actividad trofoblástica.

Se puede identificar tanto en el suero materno como en la orina por diversos procedimientos como pruebas biológicas, inmunológicas o radio-inmunoensayo.

Sus valores máximos se presentan al final del primer trimestre, encontrándose cifras que oscilan entre 60.000 y 500.000 unidades internacionales en orina de 24 horas hacia la semana 10 a 12 , descendiendo progresivamente a cifras que oscilan entre 5.000 y 20.000 unidades.

En amenaza de aborto, embarazo ectópico, disminuyen sus concentraciones; por el contrario en diabetes, eritroblastosis y toxemias se puede encontrar aumentada hacia el final de la gestación $(33,63,83)$.

\section{PROGESTERONA $Y$ PREGNANDIOL}

Durante la gestación se encuentran concentraciones crecientes de pregnandiol, con cifras normales, al término del embarazo hasta de $250 \mathrm{mg}$. en 24 horas aproximadamente.

- Su concentración no depende de la circulación fetal pues cuando hay feto muerto sigue presentándose pregnandiol en alta concentración en la orina.

- Hay grandes variaciones individuales en la excreción de pregnandiol al igual que variaciones diarias impor- tantes por lo cual su valor clínico es discutible. Además su determinación requiere técnicas complejas que hacen poco práctico el procedimiento.

La progesterona plasmática tiene valor para estudiar la función y muerte fetal pero su determinación también es difícil.

Se ha descubierto relación íntima entre la concentración de progesterona plasmática y de estriol en la orina. Cabe pensar que la estimación de progesterona plasmática pudiera brindar datos útiles. Sin embargo los métodos para estos análisis son verdaderamente complejos $(49,50,84)$.

\section{LACTOGENO PLACENTARIO HUMANO (HPL)}

Es una hormona proteínica originada en el tejido trofoblástico, aumenta progresivamente durante el embarazo pudiéndose identificar en el suero materno desde la 8 a semana de gestación (63, 131, 132).

\section{7․ CITOLOGIA EN LA EVALUACION DEL MEDIO AMBIENTE FETAL}

Papanicolaou antes de publicar sus trabajos clásicos sobre diagnóstico del cáncer del cérvix por medio de la citología, había estudiado los cambios citológicos de la vagina producidos por las diferentes influencias hormonales durante el ciclo menstrual, la menopausia y también durante el embarazo (104). Pundel ha hecho grandes contribuciones al tema (105). En la literatura médica se encuentran gran cantidad de publicaciones relacionadas con la colpocitología y el urocitograma durante el embarazo.

La citología funcional se basa en la sensibilidad del epitelio vaginal a la actividad de las hormonas sexuales, particularmente estrógenos y progesterona. 
En la evaluación del medio ambiente fetal la colpocitología nos proporciona información sobre el estado hormonal de la paciente, la microbiología del tracto genital y la presencia de atipias celulares que puede corresponder a lesiones premalignas o malignas del cuello uterino. Por lo tanto hoy se considera un procedimiento indispensable durante el control prenatal. (Fig. № 1 ).

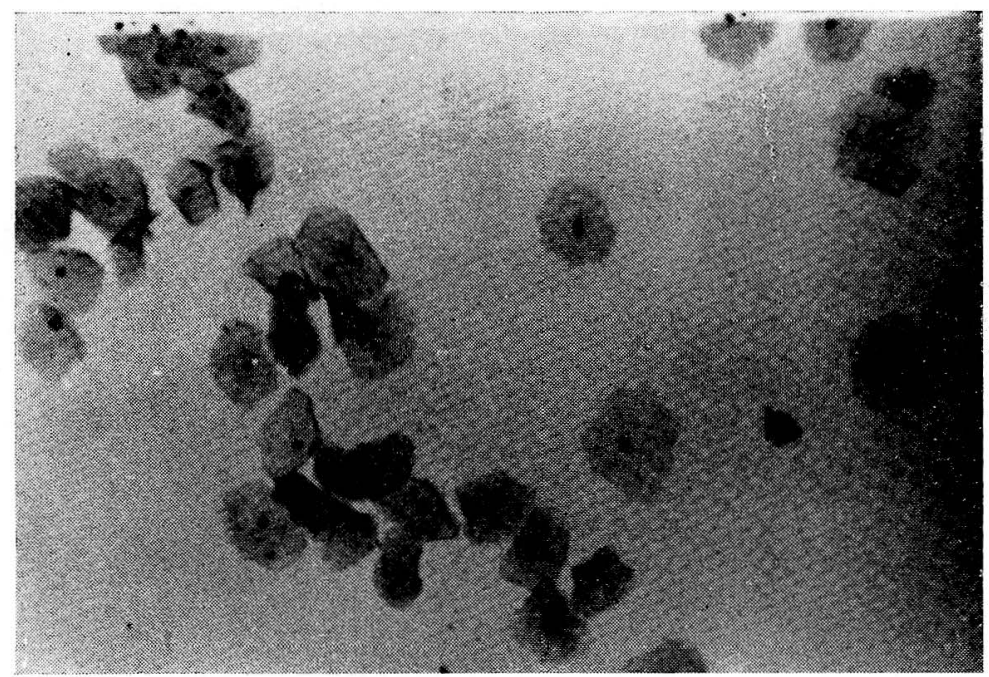

FIGURA Nọ 1 - Extendido de tipo estrogénico. Se observa en la época de la ovulación.

Las características de los frotis varían desde un extendido de tipo estrogénico en el que predominan células superficiales en el momento de la ovulación, aumentando luego las células intermedias, típicas del estímulo de progesterona, las cuales aparecen durante la gestación normal entre las semanas 2 y 14 (87, 104). (Fig. № 2). Algunos autores han informado aumento de células superficiales hacia el final de la gestación, y han tratado de relacionar este hallazgo citológico con la iniciación del trabajo de parto $(5,88)$. En las 4 a 6 semanas siguientes al parto los extendidos vaginales se caracterizan por el predominio de células parabasales, indicando ausencia de actividad tanto ovárica como placentaria (Fig. N: 3 ).
En la práctica la ctiología ha sido empleada por muchos autores con mayor o menor éxito en el manejo de pacientes con amenaza de aborto, porque en algunos de estos casos los extendidos revelan alteración del balance endocrino observándose un aumento de las células superficiales (90). En casos de feto muerto o inminencia de muerte fetal en que la placenta ha dejado de funcionar aparecen los extendidos de tipo postparto caracterizados por predominio de células parabasales. Este hallazgo ha sido también utilizado en casos de post-madurez ya que la presencia de células parabasales indican que el feto está en peligro (154).

La prueba de estrógenos administrando principalmente Dietil - Etil - 




FIGURA No 2 - Extendido de tipo progestacional caracterizado por predominio de células intermedias apelotonadas. Se observa durante el embarazo.

Bestrol en dosis que van desde 5 Mgs. hasta $50 \mathrm{Mgs}$. diarios por períodos de 5 días a una semana, ha sido recomendada por muchos auto- res, debido a que en embarazos normales los estrógenos exógenos no producen ningún cambio de tipo estrogénico en el extendido progesta-

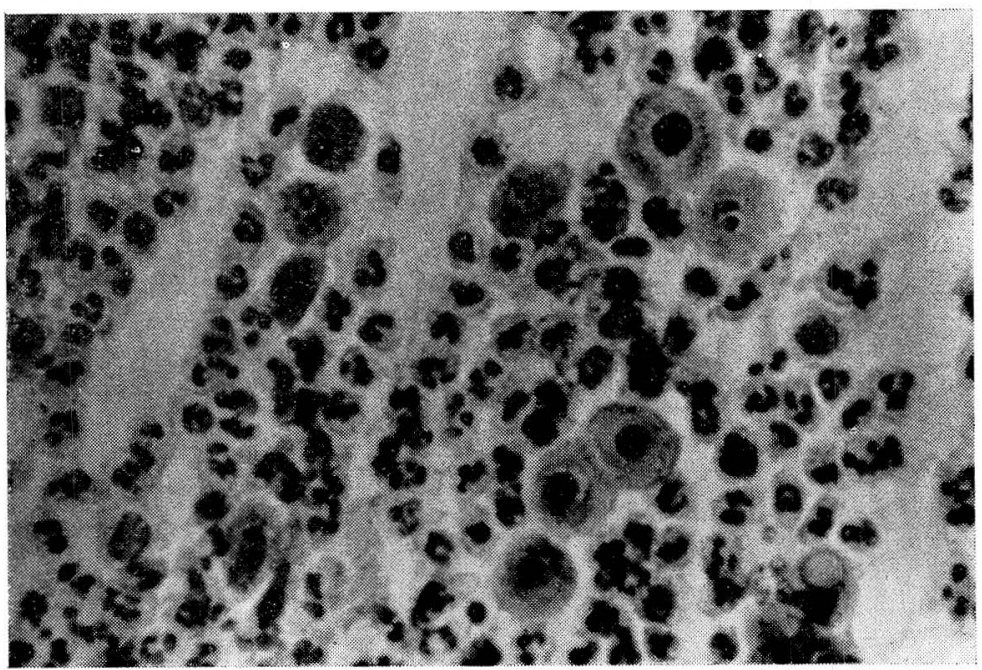

FIGURA No 3 - Extendido de tipo atrófico. Caracterizado por predominio de células parabasales. 
cional mientras que por el contrario, en amenazas de aborto por deficiencia en la producción de progesterona, o en mujeres que no están embarazadas, se va a producir un aumento significativo en el número de células superficiales de los extendidos. Esta prueba se utiliza en diagnóstico de embarazo, de amenaza de aborto, y de feto muerto $(87,150)$.

En los últimos años han aparecido publicaciones muy serias como las de Ufelder (140) sobre la presencia de Adenocarcinoma de la vagina en hijas de madres que recibieron Dietil-Etil-Bestrol durante el embarazo, por lo tanto consideramos oportuno llamar la atención sobre el peligro de emplear esta prueba durante el embarazo.

\section{ESTUDIO DEL LIQUIDO AMNIOTICO}

Su análisis ofrece un amplio campo de investigación. La recolección de datos precisos acerca de su composición se inició en el siglo pasado. Prochownick en 1877 obtuvo valores para agua, sólidos, úrea y cloruros similares a los encontrados actualmente. En 1918 Uyeno publicó un estudio amplio de la composición del líquido amniótico y añadió datos referentes a la concentración de iones inorgánicos.

Posteriormente en 1931 Makepeace (77) publicó un trabajo intitulado "A comparative study of human amniotic and maternal serum".

Cantarow (20) y Shrewsbury (116) en 1933 y Tankard (134) en 1934, contribuyeron con sendos estudios a dilucidar la composición del Líquido Amniótico.

En 1955 Hanon y Coquoin (56) revisaron la literatura sobre la compo- sición del Líquido Amniótico en una monografía intitulada "Le Liquide Amniotique". A partir de entonces los trabajos sobre líquido amniótico son numerosos y la bibliografía muy extensa.

\section{FISIOLOGIA DEL LIQUIDO AMNIOTICO}

Origen. La teoría más simplista y antigua, atribuída a Hipócrates, hablaba del líquido amniótico como el producto del riñón fetal.

Se puede decir que el líquido amniótico es un trasudado del amnios en las primeras doce semanas de la gestación. El feto y el cordón umbilical también contribuyen a su formación (101).

A partir de la semana 20 de la gestación el riñón fetal modifica sustancialmente la composición del líquido amniótico, principalmente por la presencia de productos nitrogenados. Su volumen y composición varían constantemente, calculándose su cambio total en un período aproximado de tres horas. Estos cambios están influenciados por el feto a través del riñón fetal, del pulmón, de la piel y del tracto gastrointestinal, por el cordón umbilical el cual regula el intercambio de solutos y por el amnios que contribuye tanto en la producción como en la reabsorción (44).

Antes de la semana 28 la piel fetal sirve de membrana de difusión; a partir de entonces su estratificación progresiva impide el paso de líquidos, convirtiéndose la orina fetal en la fuente principal de producción de líquido amniótico. Esto trae como consecuencia una disminución del sodio y de la osmolaridad del líquido amniótico y un aumento de productos nitrogenados $(6,10,73,74,75)$. 


\section{Volumen}

El volumen del líquido amniótico aumenta progresivamente hasta el sexto mes con un incremento promedio de $25 \mathrm{ml}$. por semana entre las semanuas 11 a 15 y de $50 \mathrm{ml}$. entre las semanas 16 a 28 . En el último trimestre hay gran variación en el volumen alcanzando su nivel máximo hacia la semana 38 , descendiendo al término alrededor de $850 \mathrm{ml}$. y aún a cantidades menores en embarazos prolongados.

Para la determinación del volumen del líquido amniótico se han utilizado diversos métodos principalmente de dilución, los cuales permiten obtener resultados muy precisos sin ningún daño para la madre o el feto. Se inyectan sustancias en el líquido amniótico tales como Rojo Congo, Rojo Fenol, Aminohipurato sódico y compuestos radioactivos como el Oxido de Deuterium y el lodo radio-activo. Estas sustancias pueden ser usadas solas o en dilución simultánea aprovechando sus diferentes características físicas y químicas y promediando los resultados obtenidos con cada una de ellas $(30,32,39,43)$.

\section{Composición}

El líquido amniótico está compuesto en su mayor parte por agua, 98 a $99 \%$ y 1 a $2 \%$ de solutos, de los cuales la mitad son sustancias orgánicas y de ellas el $50 \%$ son proteínas; el resto, componentes inorgánicos que le dan cierta semejanza con el líquido extracelular en el comienzo de la gestación $(7,74)$. Al término de ésta se hace menos parecido al líquido extracelular debido principalmente a funciones fisiológicas fetales como deglución y eliminación urinaria.

El líquido amniótico es un medio dinámico cuyos componentes sufren en su gran mayoría cambios en su concentración en relación con las diferentes edades gestacionales.

El valor promedio del $\mathrm{pH}$ es de 7.0. Su menor valor en relación con los medios con los cuales tiene intercambio (sangre fetal y materna) no ha sido explicado por ningún mecanismo exacto y se responsabiliza parcialmente a la mayor acidez de la orina fetal (44).

Diferentes autores han encontrado valores de proteínas aproximadamente 20 veces menores que las del plasma materno. En embarazos normales hasta la semana 20 a 28 la concentración de proteínas totales es relativamente alta, observándose un descenso progresivo hasta el término (4, $7,35,120,146)$.

El fibrinógeno parece estar ausente del líquido amniótico. Los aminoácidos presentan concentraciones similares a las del plasma materno.

Al comienzo de la gestación se encuentran concentraciones similares a las del plasma materno de úrea, creatinina $y$ ácido úrico $y$ al final un incremento en su concentración por el paso de la orina fetal al líquido amniótico (7, 100, 131).

Los lípidos del líquido amniótico aumentan a medida que progresa la gestación. Los fosfolípidos constituyen la fracción más importante de éstos originándose principalmente en el pulmón fetal $(54,55,58,92,125)$.

La glucosa, menor que la glicemia materna, desciende progresivamente a medida que avanza la edad gestacional. Sus valores varían entre 23 y 40 $\mathrm{mg} / 100 \mathrm{ml}$. con valores medios de $28 \mathrm{mg} / 100 \mathrm{ml}$. (121, 148). Los niveles de fosfatasa alcalina termoestable del líquido amniótico parecen incrementarse al tiempo con las del suero materno a medida que progre- 
sa el embarazo hasta el 7\% mes después del cual permanece relativamente constante. Si el líquido amniótico se contamina con meconio los niveles se aumentan considerablemente (7).

En el líquido amniótico se encuentran varios tipos de células: unas pe- queñas, redondeadas, de origen amniótico; y un segundo grupo de células descamadas de los epitelios fetales que corresponden en su mayoría a las capas del epitelio escamoso estratificado (basales, parabasales, intermedias, cornificadas - con núcleo picnótico- y anucleadas). (Fig. No $4)$.

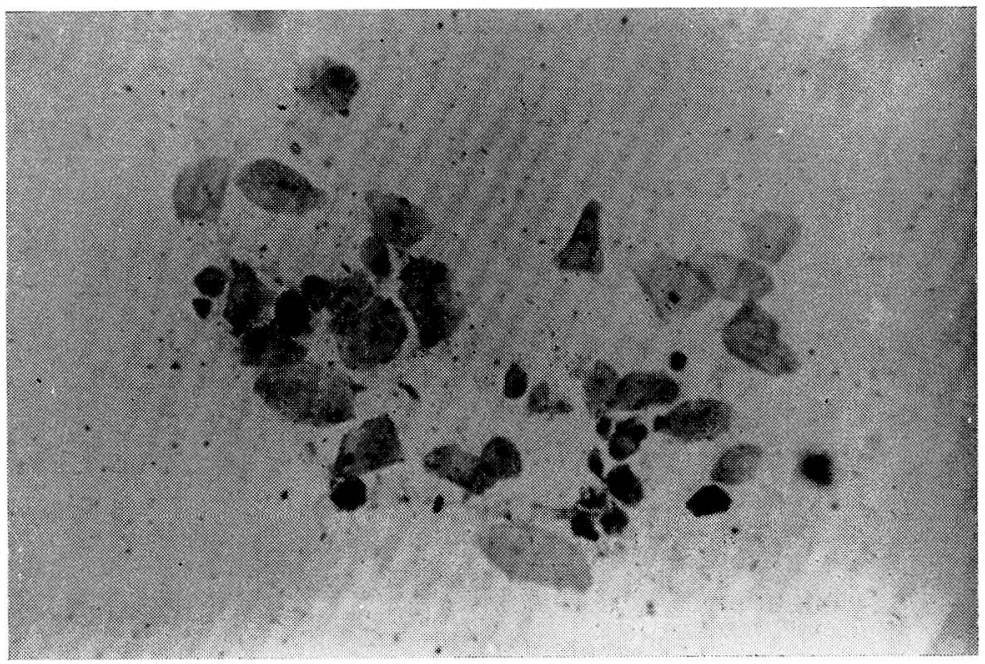

FIGURA No 4 - Células amnióticas. Las más pequeñas redondas provienen del amnios. Las otras so ndescamadas por el feto, nótese los diferentes grados de maduración celular.

Las células anucleadas y las cornificadas van aumentando progresivamente con el embarazo indicando la maduración de la piel fetal (44, 59, 75, 142). (Fotografía).

La amnioscopía ha sido también utilizada en la valoración del medio ambiente fetal; fue introducida por Saling, es un método sencillo que nos permite ver a través del cuello uterino las características macroscópicas del líquido amniótico (61).

\section{Amniocentesis}

En un principio se utilizó para evacuar el exceso de líquido amniótico en el polihidramnios.
A partir de 1937 Aburel la utilizó para la inyección de soluciones hipertónicas. En la década de los 50 Bevis (8) la empleó en el estudio de las bilirrubinas. Hacia 1960 Lilley y Freda generalizaron su empleo para el estudio de la inmunización Rh (45, 66). En los últimos 10 años la amniocentesis se ha convertido en un valioso método para la investigación del medio ambiente fetal.

\section{Indicaciones de amniocentesis}

Las múltiples indicaciones que tiene en la actualidad la práctica de este procedimiento las podemos resumir en la siguiente forma: 


\section{Genéticas}

La determinación prenatal del sexo ha sido posible utilizando la identificación del corpúsculo de Barr (28, 40, 78, 103) y más recientemente se ha complementado con la identificación del cromosoma - Y- utilizando técnicas fluorescentes con mostaza de quinacrina o hidroclorhidrato de quinacrina $(28,102,103)$. Esta determinación es importante desde el punto de vista genético para el manejo de fetos que puedan ser portadores de entidades ligadas al cromosoma $X$ como la hemofilia o la distrofia muscular de Duchene.

Con la combinación de estas dos técnicas se puede hacer un diagnóstico acertado del sexo en más del $90 \%$ de los casos.

Alteraciones congénitas del metabolismo. Estas múltiples alteraciones pueden ser trastornos en el metabolismo de los aminoácidos, de los lípidos, de los carbohidratos, de los mucopolisacáridos y otras entidades como el síndrome adreno-genital, la fibrosis quística o el xeroderma pigmentoso los cuales se pueden diagnosticar mediante el estudio de los componentes fluídos, de las células sin cultivar y de células cultivadas del líquido amniótico (41, 94, 95, 96, 97).

Aberraciones cromosómicas. Se pueden diagnosticar antes de la semana vigésima de gestación mediante el cultivo de células amnióticas elaborando cariotipo (64, 85, 86, 95, 119).

\section{Sufrimiento fetal}

Aspecto del Líquido Amniótico. La simple observación del líquido extraído por amniocentesis nos permite valorar el estado fetal: así la presencia de líquido de color amarilloso turbio la podemos encontrar en fetos Rh positivos isoinmunizados, color rojo puede indicar contaminación con sangre durante el procedimiento, color verdoso opaco es característico de la contaminación del líquido con meconio, y color de aspecto pardusco es patognomónico de muerte fetal.

Sánchez Torres en su estudio sobre embarazo prolongado describe tres tipos de líquido: abundante blanquecino con grumos de vernix presente en embarazos que cursan normalmente; un líquido abundante, sin vernix, de aspecto citrino compatible con embarazo prolongado nos debe poner alerta sobre las condiciones fetales; y finalmente líquido teñido con meconio indicativo de peligro de la vida fetal, lo cual obliga a terminar el embarazo (123).

Isoinmunización Rh. Desde que Lilley, Walker, Freda $(45,66,146)$, iniciaron estudios espectofotométricos seriados en pacientes $R h$ negativos con isoinmunización este estudio se ha convertido en una de las principales indicaciones de amniocentesis en la práctica obstétrica. Está contraindicado en mujeres embarazadas Rh. negativas con pruebas de Coombs negativa.

La dosificación de estriol en el líquido amniótico es importante en casos de embarazos complicados con anencefalia, diabetes, enfermedad renal en los cuales se han informado descensos significativos en sus concentraciones (16).

Madurez fetal. Los principales parámetros estudiados han sido: la determinación de células grasas descamadas de las glándulas sebáceas del feto, método descrito por Brosens y Gordon en 1965 utilizando sulfato de 


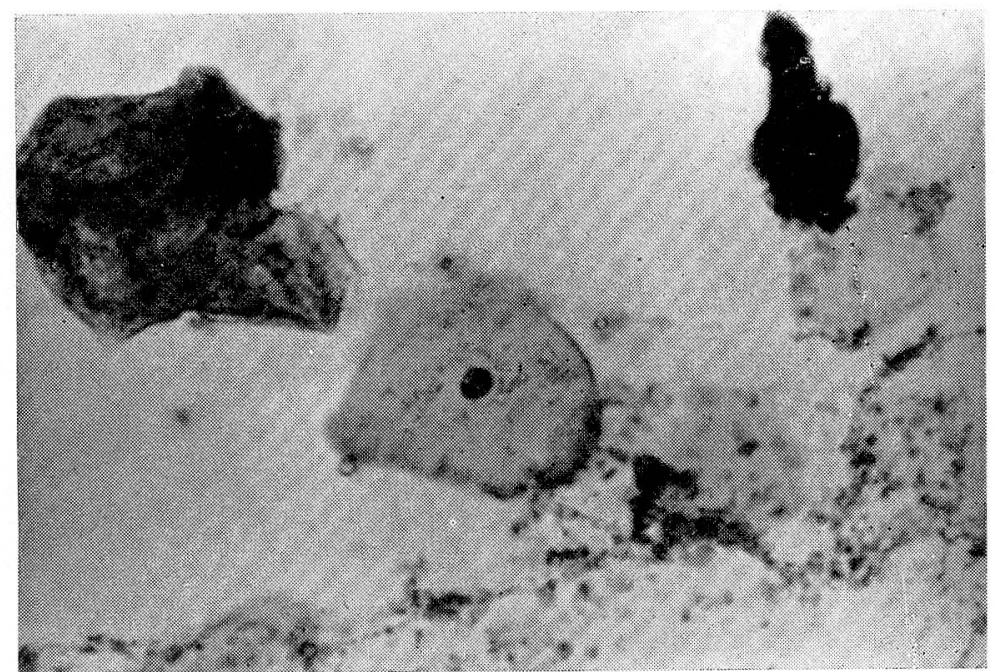

FIGURA No 5 - Células amnióticas coloreadas con sulfato de Azul de Nilo. La célula más grande anucleada se tiñe de color anaranjado.

Azul de Nilo; este método se basa en que el porcentaje de células grasas descamadas aumenta significativamente a partir de la semana 37 $(11,12,133)$. (Fig. № 5).

Pitkin (100) encontró que la concentración de creatinina en el líquido amniótico aumenta a partir de la semana 34 , y para la semana 37 los valores alcanzan cifras por encima de 2 mgs.\%.

Recientemente Gluck y cols. (54, 55) han descrito técnicas para determinar la madurez pulmonar fetal utilizando cromatografía de capa delgada para valorar la lecitina y la esfingomielina en líquido amniótico. Antes de la semana 35 los valores de estos dos fosfolípidos aumentan en forma paralela, pero a partir de la semana 35 de gestación se hace más notorio el aumento de la lecitina llegando a ser cuatro veces mayor que los de esfingomielina. La relación en la concentración de estos dos componentes son la base para la evaluación clíni- ca de la madurez de los pulmones fetales.

Otros parámetros utilizados en la estimación de madurez fetal han sido ácido úrico, úrea, proteínas, bilirrubina, $(4,9,31,81,146)$ estudios citológicos para determinar número de células poligonales anucleadas (59) o maduración celular (73).

Lind sugiere que sería mejor hablar de "desarrollo funcional del feto" en lugar del término "Madurez" y que para esta valoración deben estudiarse diferentes parámetros, idea que es compartida por otros autores (73).

\section{Indicaciones diagnósticas de amniocentesis}

Se pueden inyectar en la cavidad amniótica medios de contraste radioopacos para estudios radiológicos "Amniografía", la cual está indicada en el diagnóstico de mola hidatiforme, malformaciones fetales o muer- 
te fetal. También se pueden inyectar colorantes como índigo carmin o Rifamicina para confirmar diagnóstico de ruptura prematura de membranas, observándose la salida de líquido teñido por vagina (37); o inyectar solventes, algunos con material radioactivo, para determinación del volumen del líquido amniótico (6, 43 ).

En la monitoría fetal se utiliza la amniocentesis para registro de presiones intraamnióticas o para colocar electrodos en el feto.

\section{Indicaciones terapéuticas de amniocentesis}

Lai nyección de soluciones hiper- tónicas intra-amnióticas ha sido ampliamente utilizada en el manejo de aborto retenido y feto muerto. Como consecuencia de la legalización del aborto en muchos países este método ha despertado gran interés para interrupción de embarazos que cursan el segundo trimestre. Ultimamente se está utilizando la inyección intra-amniótica de Prostaglandina F2 Alfa con este mismo fin (18).

Otras indicaciones terapéuticas de amniocentesis son el manejo del polihidramnios agudo y la transfusión intrauterina de fetos isoinmunizados que se hallen severamente afectados (67).

\section{Segunda Parte}

\section{AMNIOCENTESIS ESTUDIO COMPARATIVO}

Con el fin de conocer en nuestro medio las dificultades técnicas, peligros y complicaciones de la Amniocentesis, la Sociedad Colombiana de Obstetricia y Ginecología coordinó un estudio cooperativo en el que participaron los Departamentos de Obstetricia y Ginecología de los hospitales docentes de Bogotá (Cuadro № 1). Nos pareció importante estudiar este aspecto porque el empleo de la amniocentesis es cada día más generalizado y aquí en Colombia todavía existe temor en la práctica de este procedimiento, y porque consi-



CUADRO No 1 
deramos esto fundamental para cualquier estudio que se haga de líquido amniótico.

Se recopiló información sobre 372 pacientes sometidas a amniocentesis, en formas especialmente elaboradas para este estudio, las cuales se transcribieron a tarjetas perforadas y los datos se procesaron en computador.
Para la punción amniótica de este estudio se seleccionaron únicamente aquellas pacientes cuyo embarazo estuviera libre de complicaciones, es decir, embarazos clasificados como de bajo riesgo y que tuvieran 260 más semanas de gestación. La edad de las pacientes estaba entre 15 y 44 años, encontrándose la mayoría entre 20 y 30 años (Cuadro № 2).

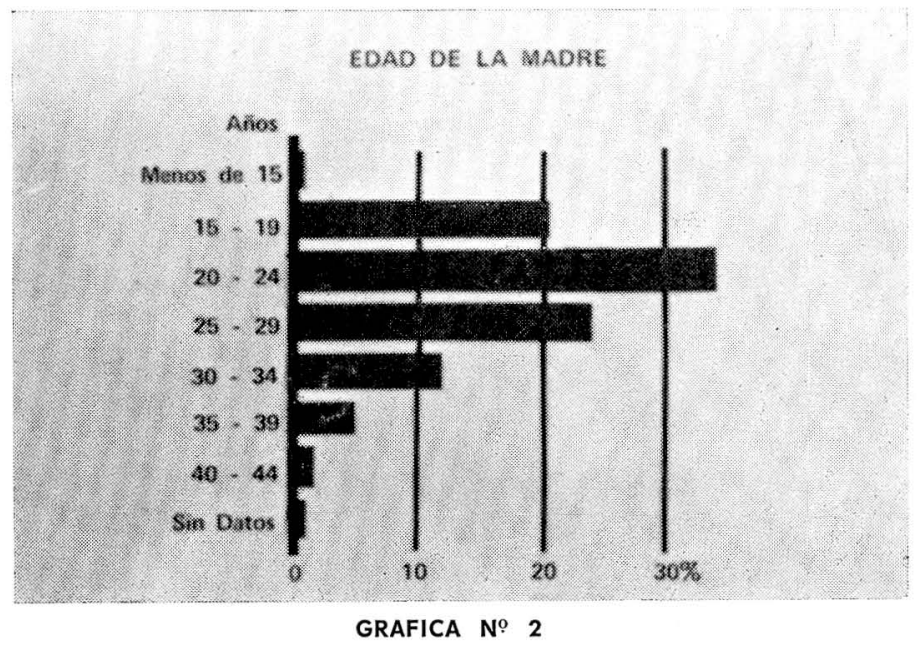

El $40 \%$ de las pacientes eran primíparas y solo seis $1.6 \%$, tenía ocho - más embarazos.

Para la codificación se agruparon las pacientes de acuerdo con la edad de gestación en grupos de dos semanas a partir de la semana 26. En el Cuadro No 3 observamos una distribución bastante uniforme en todas las semanas de gestación, así vemos como entre las semanas 26 y 41 oscilaron entre 30 y 50 pacientes y solo en el grupo de menos de 26 semanas hubo 12 y 14 en el grupo de más de 42 semanas.

Para la ejecución de la amniocentesis se colocaron las pacientes en posición decúbito dorsal, previa eva- cuación vesical, se practicó examen bimanual para determinar situación y presentación del feto, se trató de localizar placenta auscultando el "soplo placentario" en los casos en que fuera posible y se procedió a practicar una desinfección cuidadosa de la piel con jabón y soluciones antisépticas. Luego se colocó un campo estéril y la punción se efectuó con aguja de punción lumbar calibre 19 a 21. Se seleccionaron tres sitios para la punción amniótica: 1 Suprapúbica, sobre la línea media, 3 a 5 centímetros por encima del pubis rechazando la presentación hacia arriba (Fig. № 6). Esta modalidad la preferimos siempre que pudimos rechazar la presentación hacia arriba porque es la más fácil y en la que 



menos posibilidades hay de puncionar la placenta. 2 Otro sitio empleado para la amniocentesis fue el lado opuesto al dorso fetal, sobre las pequeñas partes, dos traveces de dedo por fuera de la línea media y uno - dos traveces por debajo del ombligo, y 3 detrás de la nuca fetal, cuando a la palpación se notó con certeza la sensación de líquido en esta área. (Figs. 7 y 8).

En caso de no obtener líquido o que este fuera "hemático", nunca se hicieron más de tres intentos; se desistía del procedimiento y se registraba como "punción fallida". En el $80 \%$ de los casos se obtuvo líquido en el primer intento. Nos llamó la atención la dificultad para obtener líquido en embarazos de más de 42 semanas donde fue necesario hacer dos o tres intentos en el $40 \%$ de los casos. En 16 pacientes no se obtuvo líquido después de tres intentos y se clasificaron como "punciones fallidas".

En la mayoría de las pacientes se obtuvieron entre 10 y $20 \mathrm{MI}$. de lí- 


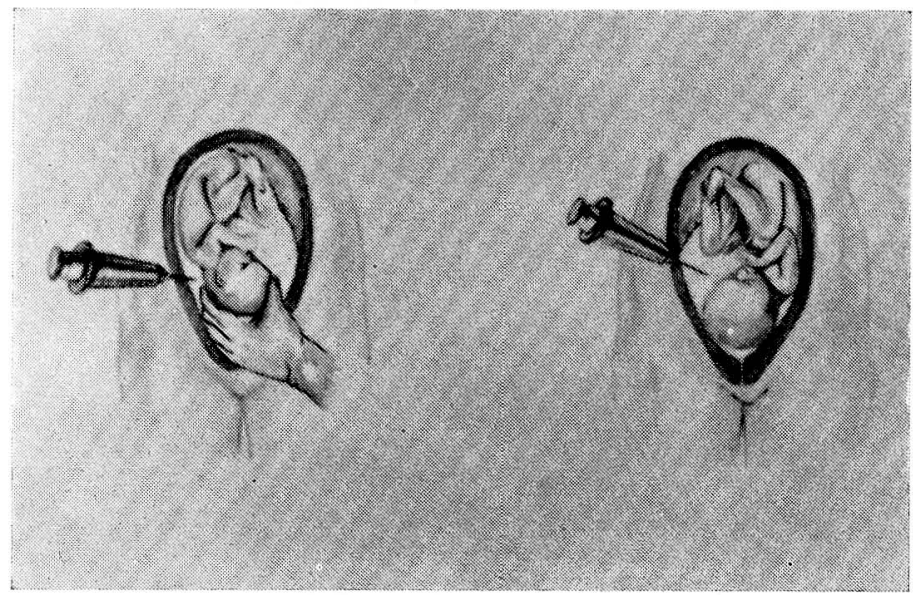

FIGURAS Nos. 7 y 8

quido amniótico. En 40 pacientes se extrajeron más de $30 \mathrm{Ml}$.

En el $75 \%$ de las pacientes se obtuvo líquido de aspecto blancuzco con grumos de vernix; líquido hemático por contaminación con sangre durante la punción en 37 casos, amarilloso en 35 casos, meconiado color verduzco en 3 casos y en 3 casos de color parduzco oscuro, los cuales correspondieron a tres pacientes con feto muerto.

La única complicación registrada fue colapso vaso-motor, la cual se presentó en 8 casos y en ninguno tuvo consecuencias para la madre o el feto. No se presentó ningún caso de desencadenamiento prematuro de trabajo de parto. El criterio seguido para analizar esta posible complicación fue considerar como trabajo de parto prematuramente aquel que se presentara dentro de las 48 horas siguientes a la amniocentesis y que el producto de la concepción pesara menos de 2.500 gramos.

En 37 casos el aspecto del líquido fue hemático, de éstos en 16 fue francamente hemático y en los 21 restantes teñido con sangre. Como en ninguno de estos casos hubo lesión fetal, no la consideramos como complicación. Ryan y cols. (114), Crystle y Rigsby (29) describen casos de lesión severa fetal por anemia o por sensibilización grave después de punción hemática y recomiendan practicar siempre que esto ocurra pruebas de laboratorio para determinar si la sangre es de origen materno o fetal y en caso de ser este último, recomiendan terminar el embarazo lo más pronto posible.

La mayoría de los autores coinciden en que las principales complicaciones de la punción amniótica son: lesión fetal directa, infección e isoinmunización. Teniendo en cuenta estos peligros consideramos contra-indicación absoluta las madres $\mathrm{Rh}$ negativas que no estén sensibilizadas $y$ aquellos casos de infección de la pared abdominal o de sepsis, y como requisitos para la punción hacemos desocupar la vejiga, observamos asepsia y antisepsia rigurosas, localizamos las partes fetales y tratamos de 
localizar la placenta. Desafortunadamente los medios adecuados para localizar placenta como ultrasonido o isótopos no los tenemos disponibles y esta es la posible causa de haber obtenido líquido hemático en 37 casos.

En ninguna de las punciones suprapúbicas obtuvimos líquido hemático, por lo tanto pensamos que es la vía de elección siempre que sea posible y tratamos de evitar el sitio de las pequeñas partes por el peligro de punción de la placenta o del cordón umbilical.

Los peligros de infección siguiendo normas estrictas de asepsia y antisepsia son mínimos. Los casos informados han sido principalmente después de inyección de soluciones hipertónicas y de transfusiones intrauterinas $(67,107,122)$.

\section{Resumen}

Se practicaron 372 amniocentesis. La única complicación registrada fue colapso vaso-motor en 8 pacientes. Hubo punción hemática en 16 pacientes y líquido teñido con sangre en 21. En ningún caso hubo lesión fetal, sin embargo llamamos la atención sobre los peligros que pueda tener la punción hemática y recomendamos la vía suprapúbica como la más segura para evitar esta eventualidad.

\section{Summary}

Three hundred and seventy-two amniocentesis were accomplished. The only complication that arose was vasomotor colapse in 8 patients. There was hematic punction in 16 patients and liquid tinted with blood in 21. There was no fetal lesion in any case; however, we call your attention to the dangers which could be caused by the hematic punction and recommend the suprapubic means as the safer to avoid this case.

\section{BIBLIOGRAFIA}

1 ALVAREZ, H. y CALDEYRO-BARCIA, R. : Contractiity of the human recorded by new methods". Surg. Gynec. Obstet. 91: 1, 1950.

2 ALVAREZ, H. Y CALDEYRO-BARCIA, R.: "Studies on the contractility of the pregnant uterus. Proc". First Wld. Congr. Fertil, Steril, Mayo 25-31, 217, 1953.

3 ALVAREZ, H. y CALDEYRO-BARCIA, R. : "La contractilidad uterina en el desprendimiento prematuro de la placenta". Arch. Gynec. Obstet. 9: 1, 1951.

4 ABBAS, T. M., y JOVEY, J. E.: "Proteins of the liquor amnii". Brit. Med. J., 1 : $476,1960$.

5 ABRAMS, R. Y., y ABRAMS, J.: "Vaginal cytology during the final weeks of pregnancy". Acta Cytol. 6: 359, 1962.

6 ABRAMOVICH, D. R.: "Fetal factors influencing volume and composition of liquor amnii". J. Obst. y Gynec. Brit. Comm. 77 : $865,1970$.

7 BONSNES, R. W.: "Composición del líquido amniótico". Clin. Obst. y Gynec. 9: 440, 1966.

8 BEVIS, D. C. A.: "Blood pigments in hemolitic disease of the new born johst". Gynec. Brit. Cwlth, 63: 68, 1956.

9 BEGNEUAND, W. P., HAWES, T. P., MICKAL, A., y SAMUELS, M.: "Amniotic fluid creatinine for prediction of fetal maturity". Obstet. Gynec. 34: 7, 1969.

10 BIEZENSKI, J. J., POMERANCE, W., y GOCDMAN, J.: "Studies on the origin of amniotic fluid lipids". Amer. J. Obstet. Gynec, 102: 853, 1968.

11 BRONSENS, T. A. : "Cytological study of amniotic fluid with nile blue sulphate staining". Acta Cytol. 10: 159, 1966.

12 BRONSENS, F. A., y GORDON: "Cytology of amniotic fluid. A new test for fetal maturity". Obstet. Gynec. 30: 652, 1967.

13 BLUTH, I.: "Estudio prenatal del feto". Clin. Obst. y Gynec. 9: 21, 1966.

14 BERNSTINE, R. L., y CALLAGAN, D. A. "Ultrasonic doppler inspection of the fetal heart". Amer. J. Obstet. Gynec. 95: 1001, 1966.

15 BABUNA, C. y YENEN, E.: "Enzimatic determination of placental function". Amer. J. Obstet, Gynec. 95: 925, 1966. 
16 BERMAN, A. M., KALCHMAN, C. G., CHATORAJ, S. C. y SCOMMEGNA, A.: "Relationship of amniotic fluid estriol to maternal urinary estriol". Amer. J. Cbstet, Gynec. 100: 15, 1968.

17 BRANDT, A. J., y BATES, J. S.: "Transabdominal amniocentesis in hydramnios". Obstet. y Ginec. 17: 392, 1961.

18 BRENNER, W., col.: "Intrauterina prostaglandin F2-Alfa for inducing abortion". Obstet. y Gynec. 39: 628, 1972.

19 BREVER, L., JOHNSON, L.: "Effects of intraamniotic injection of saline of uterine activity". Amer. J. Obstet. y Gynec. 101: 197, 1968.

20 CANTAROW, A., STUCKERT, H. and DAVIS, R. C.: Chemical composition of amniotic fluid. Surg. Gynec. Obstet. 57: 63, 1933.

21 CCBO, E., MUÑOZ, S., col.: "El embarazo de alto riesgo". Rev. Col. Obstet. y Gynec. 21: 561,1970

22 CALDEYRO-BARCIA, R.: "Uterine contractility in obstetrics". II WId. Congr. nter. Led. Obstet. y Gynec. 1: 65, 1958.

23 СCBO, E.: "Uterine hipercontractility in toxemia of pregnancy. I. its prolonged therapeutic control". Amer. J. Obstet. y Gynec. 90: 505, 1964.

24 COBO, E., QUINTERO, C. A., ESTRADA, G., y ZAMBRANO, M. D.: "Uterine behavior in abruptio placentae. I. Contraction patterns and their reactivity to ocytocin". Amer. J. Obstet. Gynec. 93: 1.151, 1965.

25 COBO, E.: "La actividad contráctil del útero humano grávido en la toxemia del embarazo". Rev. Col. Obstet. y Gynec. 14: 203, 1963

26 COHEN, B. M.: "The relationship between the fetal condition and biochemical fluid at induction of labor". J. Obstet. y Gynec. Brit. Cwlth. 71: 496, 1970.

27 COOPER, G., Jr., y CCOPER, J. B.: “Peligros de la radiación para la madre y el feto". Clin. Obstet. y Gynec. 9: 11, 1966.

28 CERVENKA, J., GORLIN, R., BENDEL, R. : "Prenatal sex determination". Obst. y Gynec. $37: 912,1971$.

29 CRYSTLE, D., RIGSBY, N. C.: "Amniocentesis experience and complications". Amer. J. Obstet. y Gynec. 106: 310, 1970.

30 CHARLES, D., JACOBY, H. E., BURGES, F.: "Amniotic fluid volumes in the second half of pregnancy". Amer. J. Obstet. y Gynec. 93: 1/042, 1965

31 CHERRY, S. H., DOLGER, H., ROSENGIELD, R., y KOCHWA, S.: "Amniotic fluir urea nitrogen, uric acid and creatinine in diabetic pregnancy". Bull. N. Y. Acad. Med. 45: 46, 1969.

32 DICKMAN, W. J., DAVIS, M. E.: "Volumetric determination of amniotic fluid with congo red". Amer. J. Obstet. y Gynec. 25: 623,1933

33 DICZFALUSY, E.: "Chorionic gonadotrophin and oestrogens in the human placenta". Acta Endoc. (Kbb), 12: Suppl. 12. 1953.

34 DICZFALUSY, E.: "Endocrinology of the fetus". Acta Obstet. Gynec. Svand. 41: (Suppl. I) 45, 1962.

35 DERRINGTON, M., y SOOTHILL, F. J.: "An inmunochemical study of the proteins of amniotic fluid and maternal and fetal serum". J. Obstet. Gynec. Brit. Comm. 68: 755, 1961

36 DUHRING, J. L., y GREENE, J. W. Jr. : "Evaluation of intrauterine fetal status". Clin. Obstet. Gynec. 9: 935, 1966.

37 DIAZ, J.: "La prueba del índigo-carmin en la ruptura prematura de membranas". Rev. Col. Obst. y Gynec. 20: 373, 1969.

38 DANDFORTH, D. N., y GORHAM, F.: "Plasma histaminase". Amer. J. Physiol. 119: 294, 1937.

39 FUCHS, F.: "Volumen del líquido amnióti$c 0$ en diversas etapas de la gestación". Clin. Obst. y Gynec. 9: 461, 1966.

40 FUCHS, F., y RIIS, P.: "Antenal sex determination". Nature, 177: 330, 1956.

41 FUCHS, F.: "Genetic information from amniotic fluid constituents". Clin. Obst. y Gynec. 9: 565, 1966.

42 FUCHS, F., y PHILIP, J.: "Possibility of antenal examination of fetal chromosomes". Nor. Med. 69: 572, 1963.

43 FUCHS, F.: "Volume of amniotic fluid at various stages of pregnancy". Clin. Obst. y Gynec. 9: 449, 1966.

44 FLOYD, W., GOODMAN, P., y WILSON, A. : "Amnictic fluid filtration and citology". Obstet. y Gynec. 34: 583, 1969.

45 FREDA, V. J.: "RH problem in obstetrics and a new concept of its management 
using amniocentesis and spectrophotometric scanning of amniotic fluid". Amer. J. Obstet. y Gynec. 92: 341, 1965.

46 FRITZ, F., CEDERWIST, L.: "Adelantos recientes en el diagnóstico prenatal por análisis del líquido amniótico". Clin. Obst. y Gynec. Marzo 1970. P. 178.

47 COLD, E. M.: "Identificación del feto que corre riesgo alto". Clin. Obstet. y Gynec. 11: 1069, 1968.

48 GOODWIN, J. W., DUNNE, J. T., THOMAS, B. W.: "Ante-partum identification of the fetus at risk". Can. Med. Assoc. J. 101 : 458, 1969.

49 GREENE, J. W., y TWEDALE, S.: "Endocrine indices of fetal enviroment". Clin. Obstet. y Gynec. 11: 1081, 1968.

50 GREENE, J. W., Jr., TWENDALE, D. E. : "Indices endocrinos del medio fetal". Clin. Obstet. y Ginec. 11: 1106, 1968.

51 GREENE, J. W., Jr., DUHRING, J. L., Y SMITH, K.: "Placental function test. $R$ review of methods available for assessment of the fetoplacental complex". Amer. J. Obstet. y Gynec. 92: 1032, 1965.

52 GREENE, J. W. Jr., SMITH, K., KYLE, G. C., TOUSCHSTONE, J. C., y DUHRING, J. L.: "Use of urinary estriol excretion in the management of pregnancies complicated by diabetes mellitus". Amer. J. Obstet. y Gynec. 91: 684, 1965.

53 GREENE, J. W., Jr., TOUCHSTONE, J. C. : "Urinary estriol as a index of placental function". Amer. J. Obstet. y Gynec. 85: $1,1963$.

54 GLUCK, L., KULOVICH, M. V., BORER, R. C., BRENNER, P. H., ANDERSON, G., SPELLACY, W. N.: "Diagnosis of the respiratory distress syndrome by amniocentesis". Amer. J. Obstet. y Gynec. 109: 440, 1971.

55 GLUCK, L., KULOVICH, M.: "Measuring the functional maturation of the fetus with the lecithin sphingomyelin ratio". Year Book Obstet. y Gynec. Pag. 256. Year Book Medical Publishers Chicago, 1972.

56 HANON, F., COQUOIN-CARNOT, M., y PIGNARD, P.: "Le liquide amniotique". Masson, Paris, 1955, p. 125.

57 HENDRICTS, C. H.: "Registro de la presión del líquido amniótico". Clin. Obstet. y Gynec. 9: 535,1966

58 HELMY, F. M., y HACK, Mñ H.: "Comparison of the lipids in maternal and cord blood and of human amniotic fluid". Proc. Soc. Exp. Bid. Med. 110: 91, 1962.

59 HUISJES, H. J.: “Cytologic features of liquor amnii". Acta Cytol. 12: 42, 1968.

60 HAWCRTH, S. G., MILIC, A. B., ADAMSON, K.: "Indices bioquímicos del estado fetal". Clin. Obstet. y Gynec. 11: 1200, 1968.

61 HENRY, G. R.: "Amnioscopy". British Journal of Hospital Medicine, Pag. 36, 1971.

$62 \mathrm{H}$. BISHOP, E.: "Vigilancia del estado fetal con métodos ultrasónicos". Clin. Obstet. y Gynec. 11: 1159, 1968.

63 JOSIMOVICH, J. B., ATWOCD, B. L.: "Human placental lactogen, a trophoblastic hormone sinengizing with chorionic gonadotrophin". Amer. J. Obstet. y Gynec. 88: 867, 1964.

64 JACOBSEN, C., BARTER, R. : "Intrauterine diagnosis and management of genetic defects". Amer. J. Obstet. y Gynec. 99: 6, 1967.

$65 \mathrm{KOHL}$, E. G.: "Comunity Obstetrical Study Progress Report. 1960. Brooklyn, New York.

66 LILEY, A. W.: "Liquor amnii analysis in the management of the pregnancy complicated by rhetsus sensibilization". Amer. J. Obstet. y Gynec. 82: 1359, 1961.

67 LILEY, A. W.: "Intrauterine transfusion of fetus in haemolitic disease". Brit. Med. J. 2: $1107,1963$.

68 LITTLEFIELD, J.: "Problems in the use of cultured amniotic fluid cells for biochemical diagnosis. Intrauterine diagnosis". 7: 5, 1971.

69 LAMBRINOPOULUS, T. C.: "Prolonged pregnancy associated with increased serum oxytocinase". Obstet. y Gynec. 23: 780, 1964.

70 LARON, Z., MANNEN HEIMER, S., y GUTHAM, S.: "Lack of transplacental passage of grouth hormone in rabits". Experientia, 22: 831, 1966.

71 LEE, A. B. H., y LEWIS, P. L.: "Alkaline phosphatas activity in normal and toxemic pregnancies". Amer. J. Obstet. y Gynec. 87: 1071, 1963.

72 LEVINE, B., ELY, C. W., y WOOD, W. A.: "Assessment of fetal maturity by maternal serum a'lkaline phosphatase analysis".

Amer. J. Obstet. y Gynec. 96: 1155, 1966.

73 LIND, T., PARKIM, F. M., y CHEYNE, G. A.: "Biochemical and cytologycal changes 
in liquor amnii with advancing gestation". J. Obstet. y Gynec. Brit. Cw/th. 76: 676, 1969

74 LIND, T., W. Z., BILLEWICZ y G. A., CHEYNE: "Composition of amniotic fluid and maternal blood through pregnancy". J. Obstet. y Gynec. Brit. Comm. 78: 505-512, 1971.

75 LIND, T., F. M., PAKIN and G. A. CHEYNE: "Biochemical and cytologic changes in amniotic fluid with advancing gestation". J. Obstet. y Gynec. Crit. Comm. 76: 673-683, 1969

76 MARMOL, J. G., VOLLAMN, R. F., GORDON, $M$. et al: "Maternal death and high risk pregnancy: An analysis of 40 maternal deaths in the collaborative project". Obstet. Gynec. 30: 816, 1967.

77 MAKREPEACE, A. W., FREEMONT-SMITH, F., DAYLEY, M. E. and CARROL, M. P. "Nature of amniotic fluid". Surg. Obstet. y Gynec. 53: 635, 1931.

78 MAKOWSKI, E. L., PREM, K. A., y KAISER, I. H.: "Detection of sex of fetuses by the incidence of sex chromatin body in nuclei cells in amniotic fluid". Science, 123 544,1956

79 MARCOU, F., ATHANASIU-VERGU, E., CHIRICEANU, D., COJMA, G., GINDOLD, N., y PARHON, C. H.: "Metabolisms of histaminase". Presse Med. Egypte, 46: 371, 1938.

80 MILLER, S. B., NAOR, E., MILKUVICH, L., y SCHMIDT, W. W.: "Serum levels of cystine - amino - peptidase, Leucine - amino peptidase, and alkaline phosphatase in single and twin pregnancies'5'. Obstet. y Gynec. 24: 707, 1964.

81 MANDELBAUM, B., LA CROIX, G. C., y ROBINSON, A. R.: "Determination of fetal maturity by spectrophotometric analysis of amniotic fluid". Obstet. y Gynec. 29: 471, 1967.

82 MESSER, R. H.: "Heat stable alkaline phosphatase as an index of placental function". Amer. J. Obstet. y Gynec. 98: 459, 1967.

83 MIDGLEY, A. R., Jr.: "Inmunoassay of human gonadotropins current status". Clin. Obstet. y Gynec. 10: 119, 1967.

84 MERKATZ, I., SOLOMON, S.: "Unidad fetoplacentaria". Clin. Obstet. y Gynec. 13: $665,1970$.

85 MILUNSKY, A., col.: "Amniocentesis para estudios genéticos prenatales". Obstet. y Gynec. 4: 1, 1973
86 MACINTYRE, N.: "Cromosomal problems of intrauterine diagnosis". Intrauterine Diagnosis. 7: 5, 1971

87 MEISELS, A.: "Citodiagnóstico hormonal durante el embarazo". Pren. Med. Mex. 30: 85, 1965.

88 MONTALVO, RUIZ L.: "Vaginal citology during delivery". Acta Cytol. 9: 337, 1965

89 NESBITT, R. Jr., AUBRY, R. H. : “High risk obstetrics 1I: Value of semiobjetive grading system in identifying the vulnerable group". Amer. J. Obstet. y Gynec. 30: 816, 1967.

90 NESBITT, Jr., R. E. L., GARCIA, R., y BONE, D. S.: "Pronostic value of vaginal cytology in pregnancy". Obstet. y Gynec. 17: 2, 1961

91 NELSON, JAMES, H., Jr.: "Nuevas fronteras en la radiología obstétrica". Clin. Obstet. y Gynec. 9: 114, 1966.

92 NELSON, G. H.: "Amniotic fluid phospholipid patherns in normal an abnormal pregnancies". Amer. J. Obstet, y Ginec. 105: 1072, 1969.

93 NERI, A., y ECKERLING, B.: "Noninfectius peritonitis: A complication of abdominal amniocentesis in acute hydramnios: Report of a case". Obstet. y Gynec. 27: 478, 1966.

94 NADLER, H. L.: "Prenatal detection of genetic defects". J. Pediatrics, 74: 132, 1969

95 NADLER, H. L.: "Antenatal detection of hereditary disorders". J. Pediatrics, 42 : 912, 1968.

96 NALLER, H.: "Intrauterine detection of genetic disorders the fetus and the newborn". Year Book, Obstet. y Gynec. 183.

97 NADLER, H.: "Indications for amniocentesis in the early prenatal detection of genetic disorders". Intrauterine Diagnosis. 7: 5, 1971.

98 OKAYAMA, T., y KOBAYASHI, Y.: "Determination of diamine oxidase activity by liquid scintillation counting". Arch. Biochem. 95: $242,1961$.

99 OJALA, A.: "Studies of bilirubin in amniotic fluid with special reference". Acta Obstet. y Gynec. Scandinav. 50: 10, 1971.

100 PITKIN, R. M. y ZWIREK, S. J.: "Amniotic fluid creatinine". Amer. J., Obstet. y Gynec. 98: 1135, 1967. 
101 PLENTL. A.: "Formation and circulation of amniotic fluid". Clin. Obstet. y Gynec. 9: 427, 1966.

102 PEARSON, P. L., BORROW, M. y VOSA C. G.: "Tecnique for identifying y chromosomes in human interphase nuclei". Nature, 226: $78,1970$.

103 PAPP, Z., GARDO, S., HERPAY, G. : "Prenatal sex determination by amniocentesis". Obstet. y Gynec. 36: 429-432, 1970.

104 PAPANICOLAOU, G. N.: "Diagnosis of early pregnancy by the vaginal smear method". Proc. Soc. Exp. Biol. (N.Y.) 22: $436,1925$.

105 PUNDEL, J. P., y VAN MEENSEL, F.: 'Gestation et citologie vaginale". Masson y Cie. Paris. 1951.

106 PENDLETON, H. J.: "Fetal heart monitoring". British Journal of Hospital Medicine, Pag. 39, 1971.

107 QLEENAN, J. T.: "Multiple intrauterine transfusion for erythroblastosis fetalis". J. Amer. Med. Ass. 191: 943, 1965.

108 QUEENAN, J. T., y WYATT, R. H.: "In,trauterine transfusion of fetus for severe erythroblaetosis fetalis". Amer. J. Obstet. y Gynec. 92: 375, 1965.

109 QUEENAN, J. T., y DOUGLAS, R. G. : “Intrauterine transfusion: A preliminary report". Obstet. y Gynec. 25: 308, 1965.

110 QUEENAN, J. T.: "Amniocentesis and transamniotic fetal transfusion for $\mathrm{Rh} \mathrm{di}$ sease". Clin. Obstet. y Gynec. 9: 491-507, 1966

111 RANTAKIALLO, P.: "Groups at risk in low birth weight infants and prenatal mortality". Acta Pediátrica Scandinávica. 193: 5, 1969

112 RIES, P., y FUCHS, F.: "Antenatal determination of fetal sex in prevention of hereditary diseases". Lancet, 2: 180, 1960.

113 RATANOSOPA, V., SCHINDLER, A. E., LEE T. Y., Y HERMANN: "Measurement of estriol in plasma by gas liquid cromatography". Amer. J. Obstet. y Gynec. 99: 295, 1957.

114 RYAN, G. T., FACO, G., ROY I. Jr. Y JACK W. PEARSON: "Fetal bleeding as a major hazard of amniocentesis". Obst. y Gynec. 40: 702-706, 1972.
115 RUSSELL, J. G.: "Obstetrics radiology". British Journal of Hospital Medicine, Pág. 23, 1971

116 SHEREWSBURY, J. F. D.: "Chemistry of the liquor amnii". Lancet 1: 415, 1933.

117 SERR, D. M., SACHS, L. y DANON, M. : "Diagnosis of sex before birth using cells from the amniotic fluid". Bull. Res. Counc. Israel, E. 5: B 137, 1955.

118 SHETTLES, L. B.: "Nuclear morphology of cells in human amniotic fluid in relation to sex of infant". Amer. J. Obstet. y Gynec. 71: 834, 1956.

119 STEELE, N. W., y BREG, W. R.: "Chromosome analysis of human amniotic fluid cells". Lancet, 1: 383, 1966.

120 SHEFF, W. F. y ESTPHAL, M.: "Fractionation of amniotic fluid proteins by zone electrophoresis". Obstet. y Gynec. 26: 826: 1965.

121 SANCHEZ, F., y ONATRU, W.: "Contenido de glucosa en el líquido amniótico de embarazos normales". Rev. Col. de Obstet. y Gynec. 24: 135, 1973 .

122 SANCHEZ, T. F.: "La amniocentesis, indicaciones técnicas y complicaciones". Rev. Fac. Med. Univ. Nal. de Col. 38: 371-377, 1972.

123 SANCHEZ, T. F.: "Amniocentesis transabdominal y embarazo prolongado". Rev. Col. Obstet. y Ginec. 13: 5, 1972.

124 SUNDEN, BERTIL: "Empleo de los ultrasonidos para el diagnóstico en Obstetricia". Rev. andorama, Pág. 10, 1969.

125 SCARPELLI, F. M.: "Thelong tracheal fluid, and lipid metabolism of the fetus". Pediatrics. 40: 951, 1967.

126 SHARP, F.: "Estimation of fetal maturity by amniotic fluid exfoliative cytology". J. Obstet. y Gynec. Brit. Cwlth. 75: 812, 1968.

127 SOUTHERN, AI. KABAYASHI Y WEINGOLD, A. B., y CARMADONY, N. C.: "Serial plasma diamino-oxidase, in first and second trimester complications of pregnancy". Amer. Obstet. y Gynec. 96: 4, 1966.

128 SOUTHERN, AI., WEINGOLD, A. B., KOBAYASHI, Y., GRIMALDI, R., GOLD, E. M. : "Diamino oxidase in diabetic pregnancy". Amer. J. Obstet. y Gynec. 101: 899, 1968., 
129 SUCKERMAN, H., SADOVSKY, B., y KALLNER, B.: "Serum alkaline phosphatase in pregnancy and puerperium". Obstet. y Gynec. 25: 819, 1965.

130 SMITH, K., GREENE, J., W. Jr., y TOUCHSTONE, J. C.: "Urinary estriol determination in the management of prolonged pregnancy". Amer. J. Cbstet. y Gynec. 96: 901, 1966.

131 SCIARRA, J. J.: "Placental protein with lactogenic and growth. Hormone like properties". Clin. Obstet. y Gynec. 10: 132, 1967.

132 SPELLACY, W. N., COHEN, W. D., y CARLSON, B. L.: "Human placental lactogen levels as a measure of placental function". Amer. J. Obstet. y Gynec. 97: 560, 1967.

133 SUAREZ, G., BUSTILLO J. Y MEDINA J.: "Estudio citológico del líquido amniótico utilizando la coloración de sulfato de azul de Nilo para determinar a madurez fetal". Rev. Col. Obst. y Ginec. XXI: 67, 1970.

134 TANKARD, A. R., BAGNALL, D. J. T., y MORRIS, F.: "Composition of the amniotic fluid analyst". 59: 806, 1934.

135 THIEDE, H. A., CREASMAN, W. I., METCAL FE, S.: "Antenatal analysis of the human chromosomes". Amer. J. Obstet. y Gynec. $94: 589,1966$

136 TITUS, M. A., REYNOLDS, B. R., GLENDENNING, M. D., and PAGE, E. W.: "Plasma aminopeptidase activity oxytocinase in pregnancy and labor". Amer. J. Obstet y Gynec. 80: 1124, 1960.

137 TRICOMI, V. G., KHL, S.: "Estado actual de la pelvimetría radiográfica". Clin. Obstet. y Gynec. 9: 44, 1966.

138 TAULOR, E. S., THOMPSON, H., GOTTESJELD, K., HOLMES, J. H., et al: "Ultrasound in obstetric and Gynecology". Amer. J., Obstet. y Gynec. 99: 671, 1967.

139 TAYLOR, E. S., THOMPSON, H., GOTTESJELD, K., HOLMES, J. H., et al: "Ultrasound diagnostic techniques in obstetricians gynecology". Amer. J. Obstet. y Gynec. 90: 655, 1964.

140 UFELDER, H.: "Current status of the stilbestrel - adenosis - carcinoma syndrome". Presentado en el VII Congreso Mundial de Obstet. y Ginec. Moscú, Agosto 1973.
141 U. S. Children's Bureau present demographic trends and their effects on maternal and child health needs and services. Washington D. C., 1968.

142 VAN LEEUWEN L., JACOBY, HANNACH, CHARLES, D.: "Exfoliative citology of amniotic fluid". Acta Citol. 9: 442, 1965.

143 VALENTI, C., KAPLAN, M.: "Diagnóstico prenatal de enfermedades hereditarias". Obstet. y Gynec. 39: 627, 1972.

144 WALLACE, H. H.: "Mortalidad y morbilidad perinatales". Clin. Obst. y Ginec. Marzo 1970. Pág. 38.

145 WALTER, A. H. C., JENNISCN, R. F.: "Antenatal prediction of hemolitic disease of newborn". Brit. Med. J., 2: 1152, 1962.

146 WILD, A. E.: "The association between protein and bilirrubin in liquor". Clin. Sci. 21: 221, 1961

147 WOYTON, J.: "Die beurteilung des reifegrades den frucht anf grunndder". $2 b l$. Gynak. 85: 552, 1963.

148 WOOD, C., ACHARYA, P. T., CORNWELL, E., y PINKERTON, J. H. M.: "The significance of glucose and lactic acid concentration in the amniotic fluid". J. Obstet. y Gynec. Brit. Cwlth. 70: 274, 1963.

149 WEINGOLD, A. B.: "Indices enzimáticos del medio fetal". Clin. Obstet. y Gynec. 11 : 1081, 1968.

150 WIED, G. L.: "Cytology during pregnan$c y$ ". Year Book of Obstet. y Gynec. Pág. 16. Year Book Medical Publishers, Chicago 1965-1966. series.

151 WIGVIST, N., BYGDEMAN, M., col.: "Current developments in the use of protaglandines for induction of abortion". Research in Prostaglandines, 2: 3, 1972.

152 WALLACE, P. G., IGNEAUD, H., col. : "Amniotic fluid creatinine for prediction of fetal maturity". Obstet. y Gynec. 34 : $1,1969$.

153 WHITE, $\mathrm{CH}$. col.: "Role and chemical and cytologic analysis of amniotic fluid in determination of fetal maturity". Amer. J. Obstet. y Gynec. 99: 6, 1967.

154 "Symposium on hormonal citology". Acta Citol. 12: 87, 1968. 